\title{
Coherent RAKE Receiver for CPM-Based Direct Sequence Spread Spectrum
}

\author{
Ke Zhou, ${ }^{1,2}$ Shilian Wang, ${ }^{1}$ and Eryang Zhang ${ }^{1}$ \\ ${ }^{1}$ Department of Electronic Science and Engineering, National University of Defense Technology, Changsha 410073, China \\ ${ }^{2}$ Institute of Electronic Engineering, China Academy of Engineering Physics, Mianyang 621900, China \\ Correspondence should be addressed to Shilian Wang; wangsl@nudt.edu.cn
}

Received 6 March 2016; Revised 20 May 2016; Accepted 29 May 2016

Academic Editor: Leonid Shaikhet

Copyright (c) $2016 \mathrm{Ke}$ Zhou et al. This is an open access article distributed under the Creative Commons Attribution License, which permits unrestricted use, distribution, and reproduction in any medium, provided the original work is properly cited.

\begin{abstract}
Direct sequence spread spectrum (DSSS) using continuous phase modulation (CPM) inherits the techniques' benefits, constant envelope, anti-interference, and spectral efficiency. To get diversity gains over a Rayleigh-fading multipath channel as in conventional direct sequence spread-spectrum binary phase shift keying (DSSS-BPSK) system, a new class of coherent RAKE receivers is proposed in this work. By introducing chip branch metric to the receiver scheme, despreading and data detection can be done meanwhile based on Maximum Likelihood Sequence Detection (MLSD). Compared to the conventional RAKE receiver which sums decision metrics symbol-by-symbol, the proposed DSSS-CPM RAKE receiver accumulates symbol branch metric increments over every phase state of multiple paths after chip phase synchronization. Numerical results show that DSSS-CPM using the synchronous despreading and demodulation algorithm has no performance loss compared to CPM system that employs MLSD algorithm under the same test conditions. Moreover, the proposed RAKE receiver outperforms conventional RAKE receiver and achieves a remarkable diversity gain of bit error rate (BER) under the Rayleigh-fading multipath channel.
\end{abstract}

\section{Introduction}

The state-of-the-art continuous phase modulation (CPM), known for its efficient spectral properties, has the merits of continuous phase and constant envelop [1], while Direct Sequence Spread Spectrum (DSSS) is a mature technique and benefits from narrowband interference suppression, low probability of intercept, and multiple-access communication [2]. The so-called DSSS-CPM signals inherit both of the technical merits specifically. Firstly, the constant envelope allows the usage of the nonlinear amplifiers which are more powerefficient and cheaper. Moreover, the narrow power spectral density (PSD) will increase the processing gain in band-limit conditions and the spread-spectrum techniques will make the signal format to have a low probability of intercept (LPI) [3]. Finally, based on the correlation properties of pseudorandom spreading sequences, a remarkable diversity gain effect can be obtained using RAKE receiver under a multipath channel and a code division multiple-access (CDMA) system is available for multiuser scenarios [4].
Historically, several types of DSSS-CPM scheme have been proposed, but most of the studies focused on modulation schemes, signal format design, multiaccess interference (MAI), and CDMA under the AWGN channel. Lok and Lehnert presented a DSSS-CPM format with continuous phase in symbol transitions as well as chip intervals [5], which is the basic scheme discussed in this paper. Because spreading sequences and data symbols are not separable, the conventional receiver structure of DSSS is not available in this DSSS-CPM system. To solve this problem, Hsu and Lehnert proposed a CDMA system. In this work, the transmitter firstly generates CPM signal of pseudorandom spreading sequence using a continuous phase modulator, and then the CPM signal is multiplied by data signal [6]. Obviously, this kind of signal is not efficient in PSD due to discontinuities at symbol intervals. Thus, McDowell proposed a dual-phase DSSS-CPM signal format $[7,8]$, which is unique from the aspect of spreading sequences and data symbols effecting the carrier phase separately. Even though this signal format is phase-continuous and despreading and dada detection 


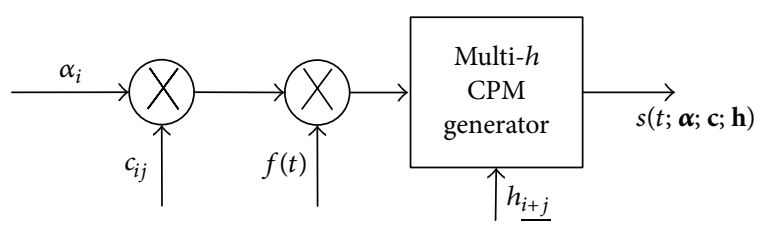

FIGURE 1: DSSS-CPM transmitter.

are separable, the data signal is limited in a minimumshift keying (MSK) format and the scheme is a compromise between performance and complexity. There are also some other researches that concentrate on spreading sequences design to eliminate the multiaccess interference (MAI) and achieve multiuser communication [9-13]. However, all of these studies did not consider RAKE receiver for DSSS-CPM system.

Diversity combining techniques were proposed because the received multipath signal will cause errors in a multipath fading channel [14]. Specifically, if the same messages over different paths are collected and recombined after phase chip synchronization, the receiver will overcome this problem and get diversity gain in performance. Conventional DSSS-PSK RAKE receiver takes advantage of pseudorandom spreading sequences to despread multipath signals and recombines the decision metrics symbol-by-symbol. This kind of RAKE receiver cannot be used in DSSS-CPM system since CPM signal is nonlinear and sequence detection is required due to the phase memory feature.

In this paper, we propose and analyze a coherent RAKE receiver for DSSS-CPM system under a Rayleigh-fading multipath channel. By introducing chip branch metric to the receiver scheme, synchronous despreading and data detection can be done based on Maximum Likelihood Sequence Detection (MLSD). The proposed RAKE receiver accumulates the symbol branch metric increments over every phase state of multiple paths after chip phase synchronization. Consequently, large diversity gains as well as desirable spectral properties can be achieved.

This paper is organized as follows. In Section 2, we describe the transmitter, the signal format, and the Rayleighfading multipath channel model. Section 3 presents the coherent RAKE receiver techniques. Some evaluations and comparisons illustrate the result in Section 4.

\section{System Model}

We describe the DSSS-CPM communication system in this section. The transmitter for this DSSS-CPM signal is presented in Section 2.1. The signal format and state trellis structure are defined in Section 2.2. Finally, in Section 2.3, we provide a model for the Rayleigh-fading multipath channel.

2.1. Transmitter. The conceptual transmitter structure of the DSSS-CPM signal is shown in Figure 1. Each information symbol is multiplied by a finite-length pseudorandom spreading sequence to form baseband spreading signals, which are then used as input to the CPM modulator. Since the signal phase is continuous throughout the symbol and chip transmissions, it inherits the desirable properties of typical CPM signals.

In the DSSS-CPM scheme, the low pass equivalent signal is defined as

$$
s(t ; \boldsymbol{\alpha} ; \mathbf{c} ; \mathbf{h})=\sqrt{\frac{2 E_{c}}{T_{c}}} \exp \{j \psi(t ; \boldsymbol{\alpha} ; \mathbf{c}, \mathbf{h})\},
$$

where $E_{c}$ is the chip energy and $T_{c}$ is the chip period. The information data $\boldsymbol{\alpha}=\left(\alpha_{0}, \alpha_{1}, \ldots\right)$ is included in the phase function which can be expressed as

$$
\psi(t ; \boldsymbol{\alpha} ; \mathbf{c} ; \mathbf{h})=2 \pi \sum_{i=0}^{\infty} \sum_{j=0}^{N_{c}-1} \alpha_{i} c_{i j} h_{i+j} q\left(t-\left(j+i N_{c}\right) T_{c}\right),
$$

where $\boldsymbol{\alpha}_{n}$ is the $M$-ary information symbol with $\boldsymbol{\alpha}_{n} \in$ $\{ \pm 1, \pm 3, \ldots, \pm(M-1)\}$ and $\mathbf{c}=\left(c_{i 0}, c_{i 1}, \ldots, c_{i N_{c}}\right) . c_{i j}$ is the pseudorandom spreading sequence with $c_{i j}= \pm 1$. $T_{s}$ is the symbol duration such that $T_{s}=N_{c} T_{c}$. The shape of the phase response can be defined as $q(t)=\int_{0}^{t} g(\tau) d \tau$. The frequency smoothing pulse $g(t)$ is limited in $\left(0, L T_{c}\right)$ for positive integer $L$. The scheme is called full-response CPM for $L=1$ and partial-response CPM for $L>1$ [9]. The modulation index $h_{(i+j) \bmod N_{h}}$ is from $\Pi=\left\{h_{0}, h_{1}, \ldots, h_{N_{h}-1}\right\}$ of cyclically varying with a fixed frequency period of $N_{h}$. The modulation index is fixed in the chip duration. The signal format is referred to Spread-Spectrum Single- $h$ CPM (SSSH-CPM) for $N_{h}=1$ and Spread-Spectrum Multi- $h$ CPM (SSMH-CPM) for $N_{h}>1$. The spreading sequence, modulation index, symbol period, and chip duration are assumed to be known a priori by both the transmitter and the intended receiver.

2.2. State Trellis Structure (Signal Format). As shown in $[2,4]$, during the $l$ th information symbol and the $n$th chip interval, the phase function can be expressed as

$$
\begin{aligned}
\psi(t ; \boldsymbol{\alpha} ; \boldsymbol{c} ; \mathbf{h})= & 2 \pi \sum_{j=n-L+1}^{n} \alpha_{l} c_{l j} h_{l+j} q\left(t-\left(j+l N_{c}\right) T_{c}\right) \\
& +\theta_{l n}=\theta\left(t, \alpha_{l}, c_{l n}, \underline{h_{l+n}}\right)+\theta_{l n},
\end{aligned}
$$

where $h_{l+n}=h_{l+n \bmod N_{h}}=k / p$ and $k$ and $p$ are fixed integers. $\theta_{l n}$, called chip accumulation phase state, can be expressed as

$$
\theta_{l n}=\left(\pi \sum_{i=0}^{l} \sum_{j=0}^{n-L} a_{i} c_{i j} \underline{h_{i+j}}\right) \bmod 2 \pi .
$$

Obviously, $\theta_{l n}$ is a Markov chain with $p$ or $2 p$ phase states, and the chip accumulated phase state $\theta_{l n}$ exhibits a periodic phase state trellis structure. If the numerator $k$ of $h_{\underline{l+n}}$ is an even number, $\theta_{l n}$ will come from a limited set as

$$
\Theta_{S}=\left\{0, \frac{\pi k}{p}, \frac{2 \pi k}{p}, \ldots, \frac{(p-1) \pi k}{p}\right\} .
$$

Otherwise, when $k$ is an odd number, then

$$
\Theta_{S}=\left\{0, \frac{\pi k}{p}, \frac{2 \pi k}{p}, \ldots, \frac{2(p-1) \pi k}{p}\right\} .
$$




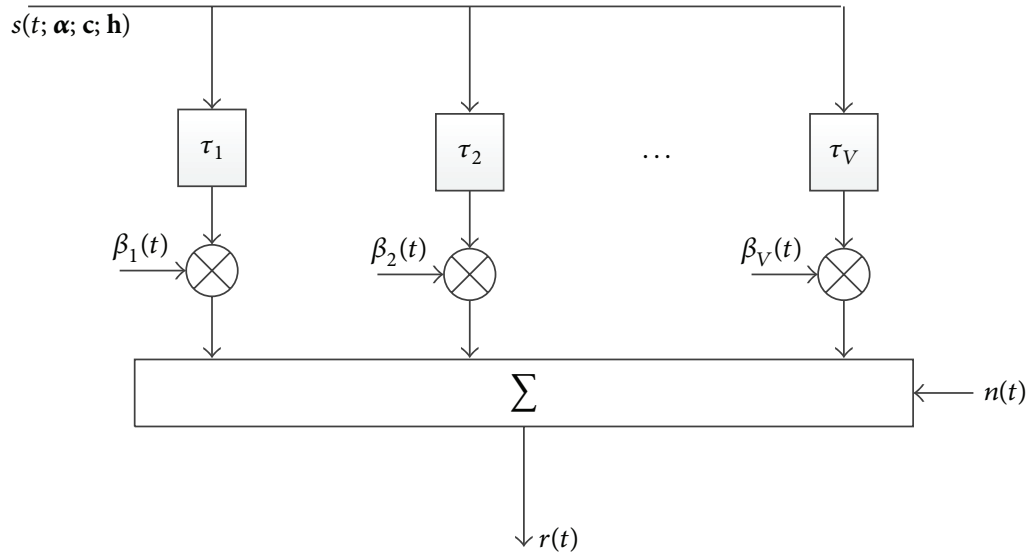

FIgURE 2: Tapped delay line channel model.

In (3), $\theta\left(t, \alpha_{l}, c_{l n}, h_{l+n}\right)$ can be expressed as

$$
\begin{aligned}
& \theta\left(t, \alpha_{l}, c_{l n}, \underline{h_{l+n}}\right) \\
& =2 \pi \alpha_{l} c_{l n} \underline{h_{l+n}} q\left(t-\left(n+l N_{c}\right) T_{c}\right) \\
& \quad+2 \pi \sum_{j=n-L+1}^{n-1} \alpha_{l} c_{l j} \underline{h_{l+j}} q\left(t-\left(j+l N_{c}\right) T_{c}\right) .
\end{aligned}
$$

The first term of (7) expresses the phase increment caused by the current $n$th chip of $l$ th symbol. And the second term indicates the phase change caused by the $L-1$ chips sequence $\left\{\alpha_{l} C_{l(n-L+1)}, \ldots, \alpha_{l} \mathcal{c}_{l(n-2)}, \alpha_{l} \mathcal{c}_{l(n-1)}\right\}$ of the $l$ th symbol $\alpha_{l}$, and it is called chip related phase state vector with $2^{L-1} M$ states.

In conclusion, at $t=\ln T_{c}$ interval of the transmission, the signaling state trellis depends on the accumulation phase state and the chip related phase state vector as

$$
\mathbf{s}_{n}=\left\{\theta_{l n}, \alpha_{l} C_{l(n-L+1)}, \ldots, \alpha_{l} \mathcal{c}_{l(n-2)}, \alpha_{l} \mathcal{c}_{l(n-1)}\right\} .
$$

At the next interval $t=l(n+1) T_{c}$, the state trellis can be expressed as

$$
\mathbf{s}_{n+1}=\left\{\theta_{l(n+1)}, \alpha_{l} \mathcal{c}_{l(n-L+2)}, \ldots, \alpha_{l} \mathcal{c}_{l(n-1)}, \alpha_{l} \mathcal{c}_{l(n)}\right\},
$$

where the accumulation phase state is recursive as

$$
\theta_{l(n+1)}=\theta_{l n}+\pi h_{\underline{l+n-L+1}} \alpha_{l} c_{l(n-L+1)}
$$

These expressions describe the phase state trellis structures which can be used for MLSD and Viterbi detection. The total states of the trellis structure are $p M \cdot 2^{L-1}$ or $p M \cdot 2^{L}$.

2.3. Channel Model. The typical features of mobile channel are multiple paths and fading due to the electromagnetic wave's reflection and scattering on buildings, trees, and other obstacles. Meanwhile, because of the movement of the receiver and obstacles, the characteristics of channel become time-varying. This section gives a Rayleigh-fading multipath channel model for the following analysis and evaluations. This tapped delay line channel model is addressed in Figure 2.
We assume that there are total $V$ paths over this channel model. Hence, the channel impulse response can be expressed as

$$
h(\tau, t)=\sum_{k=1}^{V} \beta_{k}(t) \delta\left(t-\tau_{k}(t)\right),
$$

where $\beta_{k}(t)$ is the attenuation of the $k$ th path and $\tau_{k}(t)$ is the relative time delay of the $k$ th path.

For transmitted signal $s(t ; \boldsymbol{\alpha} ; \mathbf{c} ; \mathbf{h})$ under this channel, we will receive signal

$$
r(t)=\sum_{k=1}^{V} \beta_{k}(t) s\left(t-\tau_{k}(t) ; \boldsymbol{\alpha} ; \mathbf{c} ; \mathbf{h}\right)+n(t),
$$

where $n(t)$ is white Gauss noise with a single sided spectral density of $N_{0}$.

The channel impulse response will be a zero mean Gauss process if the fading channel has large number of multiple paths. In this situation, the channel response will obey the Rayleigh distribution and the signal phase will obey a uniform distribution within $[0,2 \pi]$.

\section{Coherent RAKE Receiver}

In a synchronous DSSS-CPM system, MLSD can be used for optimum performance [2]. However, since spreading sequences and data symbols are not separable, the conventional receiver structure of the DSSS is not available in this system. To solve this problem, a coherent RAKE receiver with synchronous despreading and demodulation algorithm is derived in this section.

For frequency selective slow fading multipath channel, we assume that the attenuations and time delays are not timingvarying at least in a symbol duration, which implies $\beta_{k}(t)=$ $\beta_{k}$ and $\tau_{k}(t)=\tau_{k}$. The slow fading makes the accurate phase shift estimation possible, and consequently we can implement coherent signal detection. 
Thus, the received DSSS-CPM signal under a frequency selective slow fading multipath channel in (12) can be expressed as

$$
r(t)=\sum_{k=1}^{V} \beta_{k} s\left(t-\tau_{k} ; \boldsymbol{\alpha} ; \mathbf{c} ; \mathbf{h}\right)+n(t) .
$$

We assume that the received multipath signals are idealsynchronized and independent with each other. For convenience of expression, we use $\widetilde{x}$ and $\widehat{x}$ to indicate assumed values and estimated values.

The received signal over the $k$ th path can be expressed as

$$
r^{k}(t)=\beta_{k} s^{k}\left(t-\tau_{k} ; \boldsymbol{\alpha} ; \mathbf{c} ; \mathbf{h}\right)+n^{k}(t) .
$$

From the MLSD theory, the receiver makes a symbol decision of $\widetilde{\boldsymbol{\alpha}}_{l}$ with the principle that the received single-path signal $r^{k}(t)$ and the assumed waveform $s^{k}\left(t ; \widetilde{\boldsymbol{\alpha}}_{i} ; \mathbf{c} ; \mathbf{h}\right)$ have the minimum squared distance as

$$
\lambda^{k}(\widetilde{\boldsymbol{\alpha}})=\int_{-\infty}^{\infty}\left|r^{k}(t)-s^{k}(t ; \tilde{\boldsymbol{\alpha}} ; \mathbf{c} ; \mathbf{h})\right|^{2} d t .
$$

Due to the constant envelope of DSSS-CPM signal, minimum equation (15) is equivalent to the maximum crosscorrelation, given by

$$
\lambda^{k}(\widehat{\alpha})=\operatorname{Re}\left[\int_{-\infty}^{\infty} r^{k}(t) s^{k}(t ; \widetilde{\boldsymbol{\alpha}} ; \mathbf{c} ; \mathbf{h})^{*} d t\right] .
$$

Using Viterbi algorithm [2], the right side of (16) can be expressed as

$$
\begin{aligned}
\lambda_{i}^{k}(n)= & \lambda_{i}^{k}(n-1) \\
& +\operatorname{Re} \underbrace{\left[\int_{n T_{s}}^{(n+1) T_{s}} r^{k}(t) s^{k}(t ; \tilde{\boldsymbol{\alpha}} ; \mathbf{c} ; \mathbf{h})^{*} d t\right]}_{\Delta \lambda_{i}^{k}(n)},
\end{aligned}
$$

where the first term, $\lambda_{i}^{k}(n-1)$, is the branch metric of the $i$ th survive path of state trellis over the $k$ th multipath at $t=$ $(n-1) T_{s}$ and the second term which is called symbol branch metric increment presents the metric increment caused by the $n$th possible symbol $\widetilde{\boldsymbol{\alpha}}_{n}$.

With the assumed symbol $\widetilde{\boldsymbol{\alpha}}_{n}$ and the spreading sequence $\left\{\widetilde{\alpha}_{n} c_{n(1)}, \ldots, \widetilde{\alpha}_{n} c_{n\left(N_{c}-1\right)}, \widetilde{\alpha}_{n} c_{n\left(N_{c}\right)}\right\}$, the symbol branch metric increment can be written in the following manner:

$$
\begin{array}{r}
\Delta \lambda_{i}^{k}(n)=\operatorname{Re}\left[\int_{m T_{c}}^{\left(m+N_{c}\right) T_{c}} r^{k}(t) s^{k}(t ; \tilde{\boldsymbol{\alpha}} ; \mathbf{c} ; \mathbf{h})^{*} d t\right] \\
=\sum_{j=0}^{N_{c}-1} \underbrace{\operatorname{Re}\left[\int_{n T_{s}+j T_{c}}^{n T_{s}+(j+1) T_{c}} r^{k}(t) s^{k}(t ; \tilde{\boldsymbol{\alpha}} ; \mathbf{c} ; \mathbf{h})^{*} d t\right]}_{\Lambda_{n}^{k}(j)},
\end{array}
$$

where $\Lambda_{n}^{k}(j)$, called chip branch metric increment, indicates the chip branch metric caused by the $j$ th possible chip $\widetilde{\alpha}_{n} c_{n(j)}$. Thus, we have the following equation: $\Delta \lambda_{i}^{k}(n)=\sum_{j=0}^{N_{c}-1} \Lambda_{n}^{k}(j)$.
As presented in Section 2, the chip accumulated phase state $\theta_{l n}$ is from a limited set $\Theta_{S}$. Thus, the chip branch metric increment can be expressed as

$$
\begin{aligned}
& \Lambda_{n}^{k}(j) \\
& \quad=\operatorname{Re}\left[e^{-j \theta_{n} j} \int_{n T_{s}+j T_{c}}^{n T_{s}+(j+1) T_{c}} r^{k}(t) e^{-j \theta\left(t, \widetilde{\alpha}_{n}, c_{n j}, h_{n+j}\right)} d t\right],
\end{aligned}
$$

where $e^{-j \theta_{n j}}$ forms a limited phase rotation network. With (18) and (19), we can now rewrite (17) as

$$
\lambda_{i}^{k}(n)=\lambda_{i}^{k}(n-1)+\sum_{j=0}^{N_{c}-1} \Lambda_{n}^{k}(j) .
$$

These expressions indicate that the decision metrics of the $k$ th path form a Markov chain and a Viterbi decoder can be used for demodulating the information symbols of independent paths under a Rayleigh-fading multipath channel.

Thus, we have the decision metrics, and the next step is to implement a RAKE receiver to get diversity gain over multipath channel. A normal idea is combining the metrics before symbol decision, but it will not work Since DSSSCPM signal format is nonlinear and MLSD algorithm is a sequence decision method, different from the conventional RAKE receiver computing the decision metrics symbol-bysymbol, we proposed a coherent RAKE receiver for DSSSCPM system, which accumulates the symbol branch metric increment in every phase state of the trellis structure over multiple paths instead. Based on this idea, the RAKE receiver synchronizes the chip phase of the main separated $G$ paths and combines the symbol branch metric increments, where $G$ is the number of main separated paths that we choose based on principle of minimizing the BER and acceptable hardware complexity. The total symbol branch metric increment can be expressed as

$$
\Delta \lambda_{i}(n)=\sum_{k=1}^{G} \gamma_{k} \Delta \lambda_{i}^{k}(n)=\sum_{k=1}^{G} \sum_{j=0}^{N_{c}-1} \gamma_{k} \Lambda_{n}^{k}(j),
$$

where $\gamma_{k}$ is the metric weighting coefficient of the $k$ th path, which is defined as

$$
\gamma_{k}=\frac{\beta_{k}}{\sum_{s=1}^{G} \beta_{s}} .
$$

This combination method is called maximum ratio combining (MRC). The larger the path attenuation is, the less contribution is made to the total symbol branch metric increment.

The total symbol branch metric of the $i$ th survive path in phase state trellis structure can be expressed as

$$
\lambda_{i}(n)=\lambda_{i}(n-1)+\sum_{k=1}^{G} \sum_{j=0}^{N_{c}-1} \gamma_{k} \Lambda_{n}^{k}(j) .
$$

Equation (23) illuminates that decision metrics are the weighted sum of chip branch metric increments over every 


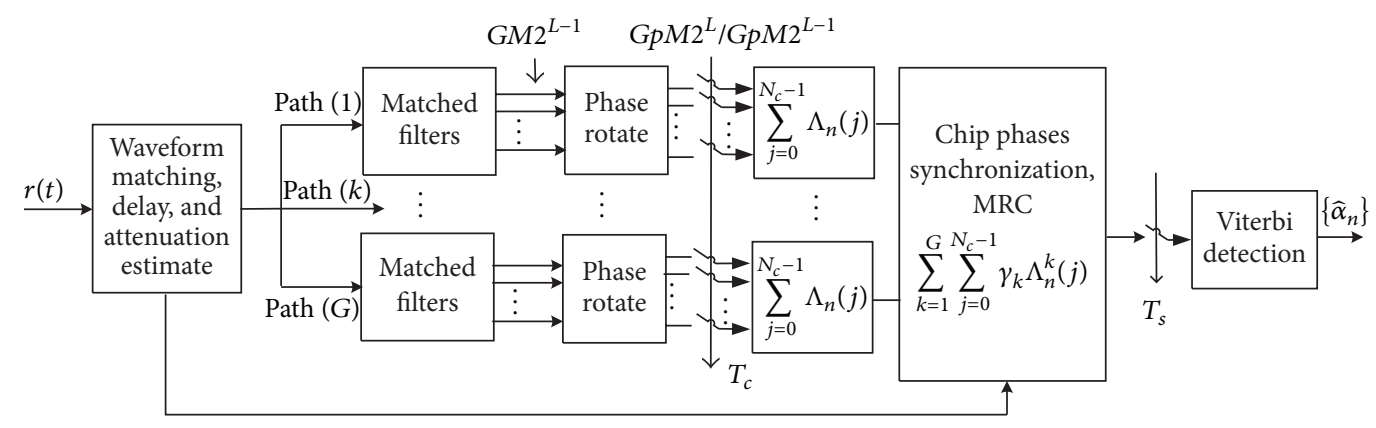

FIGURE 3: Coherent RAKE receiver for DSSS-CPM.

phase state. Using the branch metric and Viterbi decoder algorithm, the conceptual structure of coherent RAKE receiver architecture for DSSS-CPM is shown in Figure 3.

Standard DSSS-BPSK system takes advantage of orthogonal spreading sequences to synchronize with local spreading codes and obtain multipath time delay and attenuation. However, the DSSS-CPM signal is such that the transmitted signals have continuous phase. Hence, it is difficult to take use of the orthogonality of spreading sequences to distinguish the multipath signals as in DSSS-BPSK system. As a possible solution, the waveform-matching block, as shown in Figure 3, is used to distinguish multipaths and estimating the multipath time delay and attenuation. The received signal is firstly moved to match with the CPM waveform generated by the chosen spreading sequence using the same CPM modulation parameters as the transmitter. According to the waveformmatching correlation peaks, the receiver can obtain the delays and relative attenuations of main paths.

The matched filters block calculates chip branch metric increment according to (19), which calculate the correlation of the $k$ th path signal $r^{k}(t)$ with the assumed local waveform $s^{k}\left(t ; \widetilde{\boldsymbol{\alpha}}_{i} ; \mathbf{c} ; \mathbf{h}\right)$. The correlation is then multiplied by the phase rotation network $e^{-j \theta_{n j}}$ in phase rotate block.

We have calculated that the total phase state of the trellis structure is $p M \cdot 2^{L-1}$ or $p M \cdot 2^{L}$ over one path of the channel. In order to obtain the complete chip branch metric increments $\Lambda_{n}^{k}(j)$ for $k=1,2, \ldots, G$, the RAKE receiver needs a bank of $G p M \cdot 2^{L-1}$ or GpM $\cdot 2^{L}$ matched correlation filters to match with local waveforms $s^{k}\left(t ; \widetilde{\boldsymbol{\alpha}}_{i} ; \mathbf{c} ; \mathbf{h}\right)$ for $k=1,2, \ldots, G$, as is shown in Figure 3. Obviously, the hardware complexity increases with diversity level $G$, modulation level $M$, and memory length $L$. Hence, the compromise between complexity and performance should be taken into consideration in an actual DSSS-CPM system.

\section{Numerical Examples}

In this section, we numerically present the simulation results of synchronous despreading and demodulation and coherent RAKE receiver for DSSS-CPM system. In Section 4.1, we validate the feasibility of the coherent receiver algorithm for SSSH-CPM and SSMH-CPM. BER performance results of coherent RAKE receiver for DSSS-CPM under a Rayleighfading multipath channel are presented in Section 4.2.

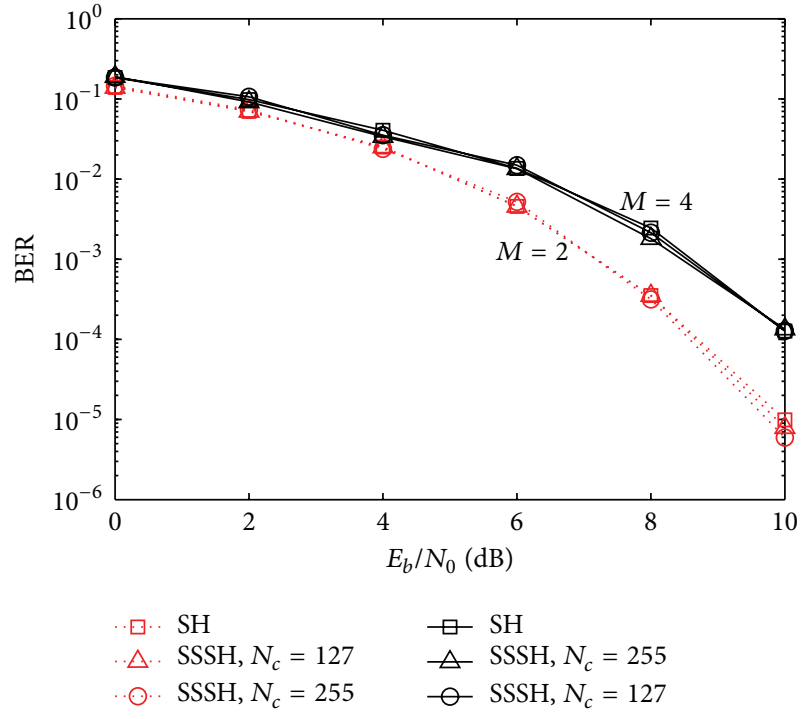

FIGURE 4: BER performance of SSSH-CPM in an AWGN channel $(L=1, h=1 / 2)$.

Moreover, a comparison is made to DSSS-BPSK which employs a conventional RAKE receiver. In the following examples, $m$-sequences generated by a linear shift register and raised cosine pulses are used to determine the performance. We assume that the symbol, the carrier, and the pseudorandom spreading sequence are synchronized perfectly. The parameters of the multipath channel are not timing-varying at least in a symbol interval. The following work is completed in MATLAB.

4.1. BER of Synchronous Despreading and Demodulation. While significant study has been done on general CPM signaling format, a BER performance comparison is made to the optimal receiver based on MLSD in an AWGN channel.

In Figures 4-5, we show BER results for SSSH-CPM system and SSMH-CPM system with different modulation parameter values. "SH" and "MH" indicate single- $h \mathrm{CPM}$ and multi- $h$ CPM. The curves reflect that the BER performances of DSSS-CPM system with $N_{c}=127$ and $N_{c}=$ 255 are coincident when other parameter values are the same. Moreover, there is almost no performance loss in 


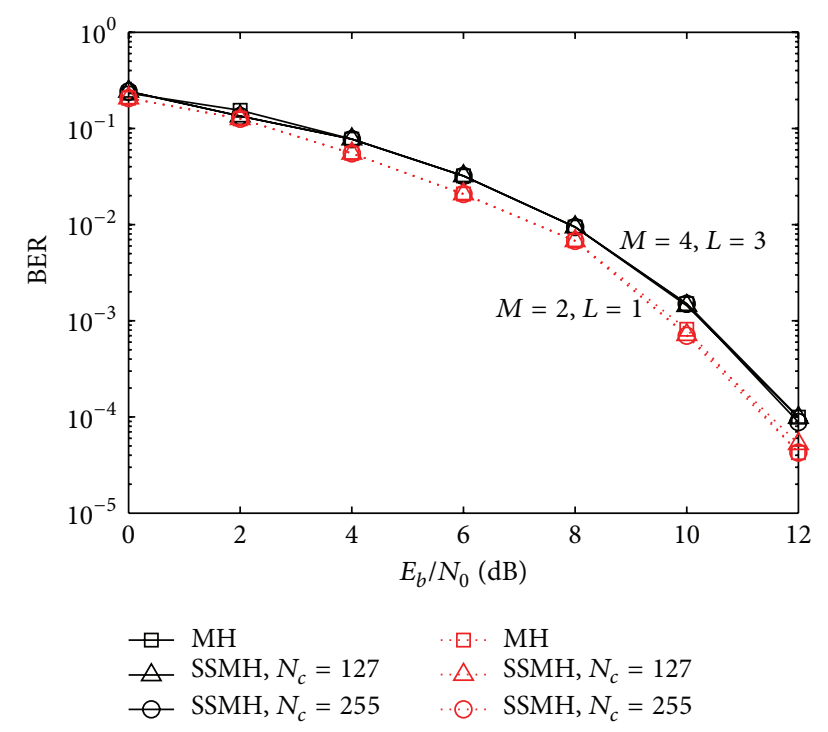

FIGURE 5: BER performance of SSMH-CPM in an AWGN channel $(h=[1 / 4,2 / 4])$.

SSSH-CPM and SSMH-CPM compared to SH-CPM and MH-CPM which employ optimal receiver based on MLSD.

These examples and analysis reflect that the synchronous despreading and demodulation receiver based on MLSD presented in the last section is effective for all kinds of DSSSCPM, including SSSH-CPM, SSMH-CPM, full-response DSSS-CPM, partial-response DSSS-CPM, and two- or fourlevel DSSS-CPM.

4.2. Performance of Coherent RAKE Receiver. There are a large number of multipath components in an actual multipath scattering channel (e.g., ionosphere and troposphere scattering), but we can obtain appropriate diversity gain and suitable hardware complexity with $G=2 \sim 3$ in the most multipath conditions [14]. According to typical cellular and microwave environment, the following simulation samples use relative attenuations $[0,-5 \mathrm{~dB},-10 \mathrm{~dB}]$, which are the maximum energy paths of the totally $V$ paths over the Rayleigh-fading multipath channel model presented in Section 2.3. The relative delay time of the three paths is $\left[0,16 T_{\text {stp }}, 32 T_{\text {stp }}\right]$, where the temporal resolution is defined as $T_{\text {stp }}=T_{s} /\left(N_{c} * N_{\text {samp }}\right)$ and the sampling rate is set to $N_{\text {samp }}=4$. Hence, the relative delay time of the 3 paths can be rewritten as $\left[0,4 T_{c}, 8 T_{c}\right]$. The multipath signal phases obey a uniform distribution within $[0,2 \pi]$. Set the normalized Doppler frequency as $f_{d}=0.01 \mathrm{~Hz}$, which means the multipath channel is slow fading.

The received signal is firstly moved to match with the $\mathrm{CPM}$ waveform generated by the spreading sequence as shown in the first block of Figure 3. We can then obtain the time delays and the relative attenuations of the paths that have larger energy according to these waveform-matching correlation peaks as shown in Figure 6. Moreover, it is concluded that the multipath time delay temporal resolution is less than a chip interval. So the receiver can add up the chip branch metric increments of main paths within the

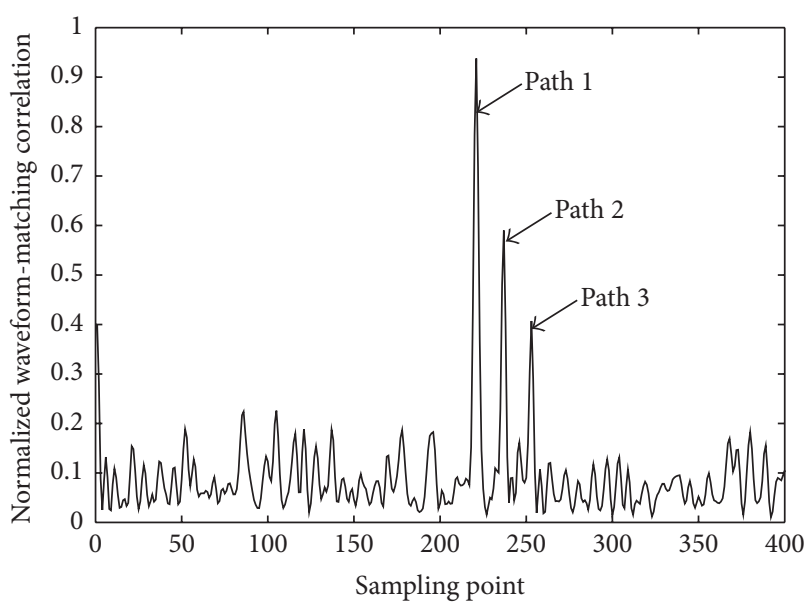

FIGURE 6: Normalized waveform-matching correlation peaks.

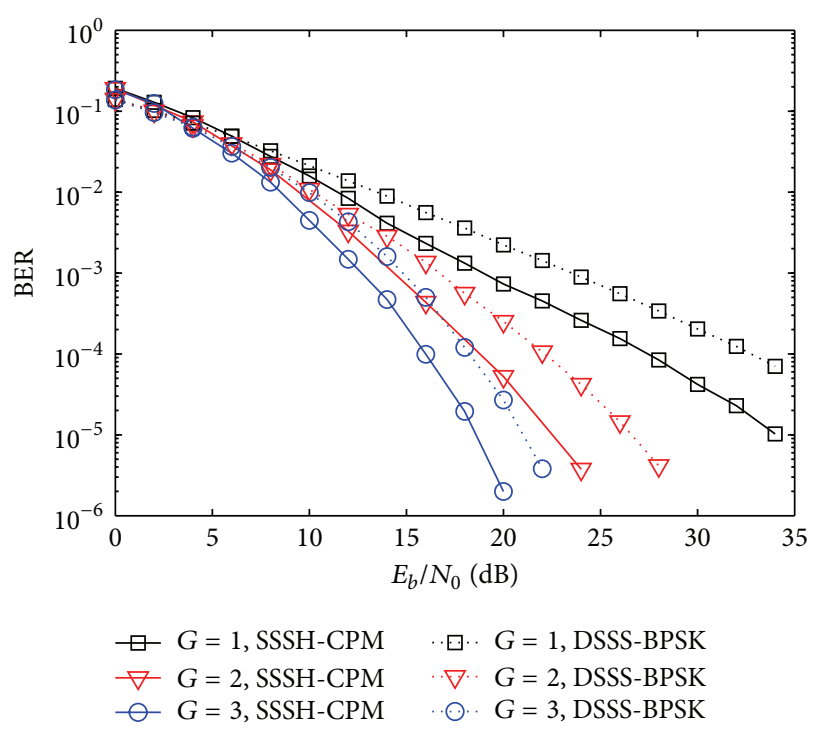

FIGURE 7: BER curves of the SSSH-CPM and DSSS-BPSK using RAKE receiver $\left(M=2, L=1, h=1 / 2\right.$, and $\left.N_{c}=63\right)$.

chip duration according to the estimation of time delays and attenuations.

The probability curves of BER for DSSS-CPM using the proposed RAKE receiver in a Rayleigh-fading multipath channel are shown in Figures 7 and 8. Besides, Figure 7 also shows the BER performance curves of a standard DSSSBPSK system using a conventional RAKE receiver with the same symbol duration $T_{s}$ and the same $m$-sequence for comparison. $G=1$ indicates the BER performance without diversity gain and the receiver uses only one path to make symbol decisions. $G=2,3$ means two- or three-level diversity. Due to the memory characteristic of DSSS-CPM signal format, SSSH-CPM outperforms the conventional DSSS-BPSK system at high signal-to-noise ratio. There are about $4 \mathrm{~dB}$ and $3 \mathrm{~dB}$ improvement of performance at BER = $10^{-5}$ for two- and three-level diversity compared to DSSSBPSK in the same test conditions. It is also observed from 


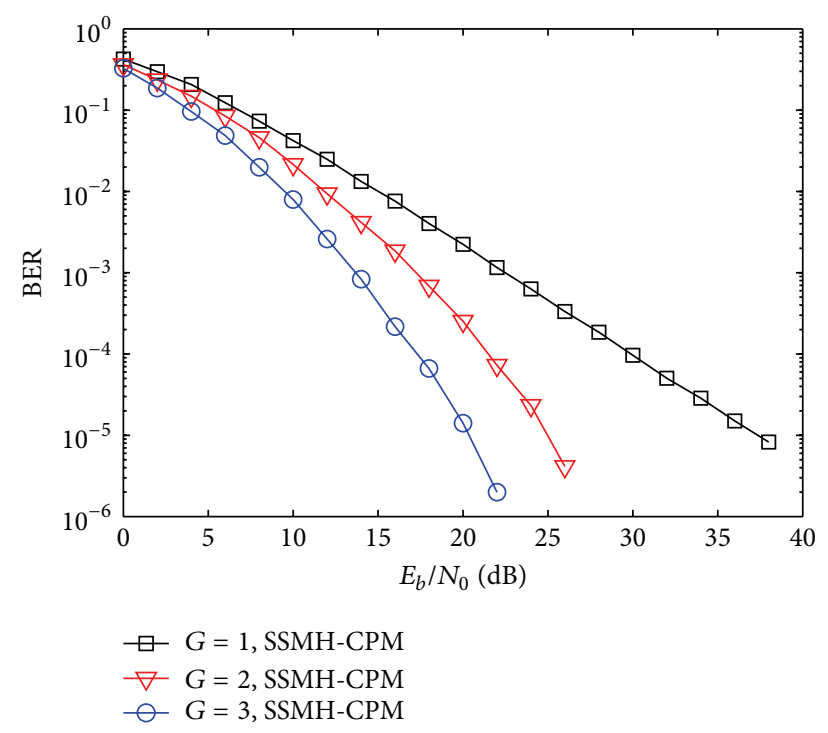

FIGURE 8: BER curves of the SSMH-CPM using RAKE receiver $(M=$ $4, L=2, h=[1 / 4,2 / 4]$, and $\left.N_{c}=63\right)$.

Figure 7 that the proposed RAKE receiver has remarkable performance gain while achieving $12 \mathrm{~dB}$ and $16 \mathrm{~dB}$ of diversity gain at BER $=10^{-5}$ for $G=2,3$ considered in this paper. Similar diversity gain for SSMH-CPM is shown in Figure 8.

These numerical results show that performance improvement can be achieved over conventional DSSS-BPSK RAKE receiver. Furthermore, the proposed RAKE receiver can obtain a remarkable diversity gain, while the DSSS-CPM signaling format maintains constant envelope and spectral efficiency.

\section{Conclusions}

Motivated by the excellent properties of DSSS-CPM communication system, a new class of coherent RAKE receiver based on MLSD has been proposed in this paper. By introducing chip branch metric increment to the receiver scheme, the synchronous despreading and demodulation algorithm and the coherent RAKE receiver are presented for DSSSCPM system. We have provided numerical examples to validate the efficiency of the algorithm. It shows that there is almost no performance loss in SSSH-CPM and SSMH-CPM compared with simple CPM system employing an optimal receiver. Moreover, SSSH-CPM significantly outperforms conventional standard DSSS-BPSK under a Rayleigh-fading multipath channel, and remarkable diversity gains can be achieved in DSSS-CPM system using the proposed RAKE receiver.

\section{Competing Interests}

The authors declare that there are no competing interests regarding the publication of this paper.

\section{References}

[1] C.-E. Sundberg, “Continuous phase modulation," IEEE Communications Magazine, vol. 24, no. 4, pp. 25-38, 1986.

[2] W. D. Lane and A. M. Bush, "Spread-spectrum multi-h modulation," IEEE Journal on Selected Areas in Communications, vol. 8, no. 5, pp. 728-742, 1990.

[3] D. K. Asano, T. Hayashi, and R. Kohno, "Modulation and processing gain tradeoffs in DS-CDMA spread spectrum systems," in Proceedings of the IEEE 5th International Symposium on Spread Spectrum Techniques and Applications, vol. 1, pp. 9-13, 1998.

[4] R. T. Hsu and J. S. Lehnert, "Continuous phase-coded directsequence spread-spectrum multiple-access communications," in Proceedings of the 10th Annual International Phoenix Conference on Computers and Communications, pp. 441-447, March 1991.

[5] T. M. Lok and J. S. Lehnert, "DS/SSMA communication system with trellis coding and CPM," IEEE Journal on Selected Areas in Communications, vol. 12, no. 4, pp. 716-722, 1994.

[6] R. T. Hsu and J. S. Lehnert, "The performance of continuousphase-coded DS/SSMA communications," IEEE Transactions on Communications, vol. 46, no. 4, pp. 533-543, 1998.

[7] A. T. McDowell, J. S. Lehnert, and Y. K. Jeong, "Dual-phase continuous phase modulation for spread-spectrum multipleaccess communication," IEEE Transactions on Communications, vol. 52, no. 5, pp. 823-833, 2004.

[8] A. T. McDowell and J. S. Lehnert, "Phase-independent continuous phase modulation for bandwidth efficient multipleaccess communication," in Proceedings of the IEEE Military Communications Conference (MILCOM '92) Communications, Fusing Command, Control and Intelligence, vol. 1, pp. 104-107, IEEE, San Diego, Calif, USA, 1992.

[9] R. R. Müller and A. Lampe, "Spectral efficiency of random CDMA with constant envelope modulation," AEUInternational Journal of Electronics and Communications, vol. 65, no. 8, pp. 701-706, 2011.

[10] J. S. Lehnert, "Serial MSK spread-spectrum multiple-access communications," IEEE Transactions on Communications, vol. 40, no. 6, pp. 1119-1127, 1992.

[11] R. R. Müller, "On random CDMA with constant envelope," in Proceedings of the IEEE International Symposium on Information Theory (ISIT '11), pp. 1663-1667, St. Petersburg, Russia, August 2011.

[12] N. Mazzali, G. Colavolpe, and S. Buzzi, "CPM-based spread spectrum systems for multi-user communications," IEEE Transactions on Wireless Communications, vol. 12, no. 1, pp. 358-367, 2013.

[13] Y. Fengfan, H. Leung, B. Guangguo, and Y. Ming, "The design criterion of novel phase spreading sequences for mobile DS/SSMA communications," in Proceedings of the IEEE Sixth International Symposium on Spread Spectrum Techniques and Applications, vol. 1, pp. 207-211, Parsippany, NJ, USA, September 2000.

[14] G. T. Chyi, J. G. Proakis, and C. M. Keller, "Diversity selection/combining schemes with excess noise-only diversity reception over a rayleigh-fading multipath channel," in Proceedings of the Conference on Information Sciences and Systems (CISS '88), Princeton University, March 1988. 


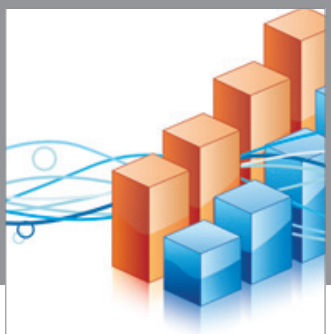

Advances in

Operations Research

vatem alat4

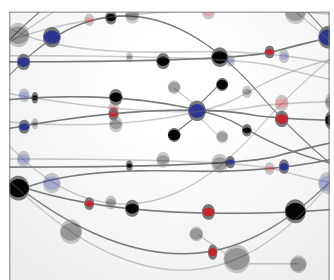

\section{The Scientific} World Journal
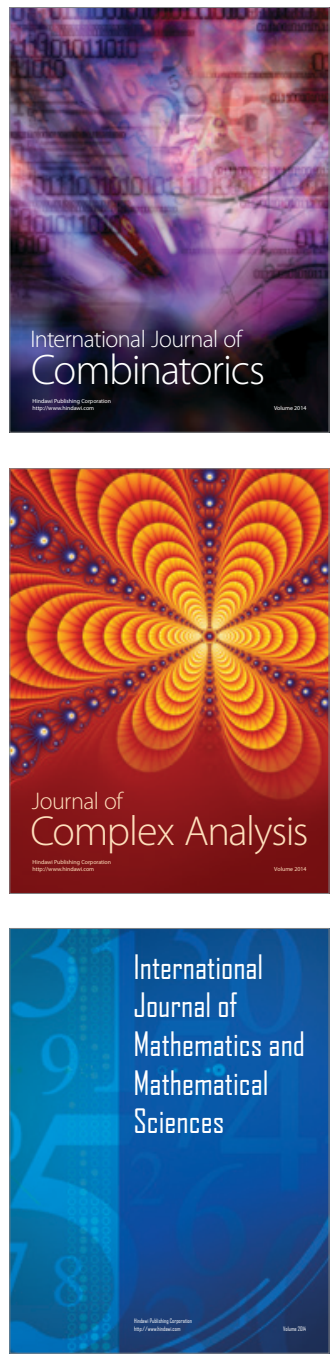
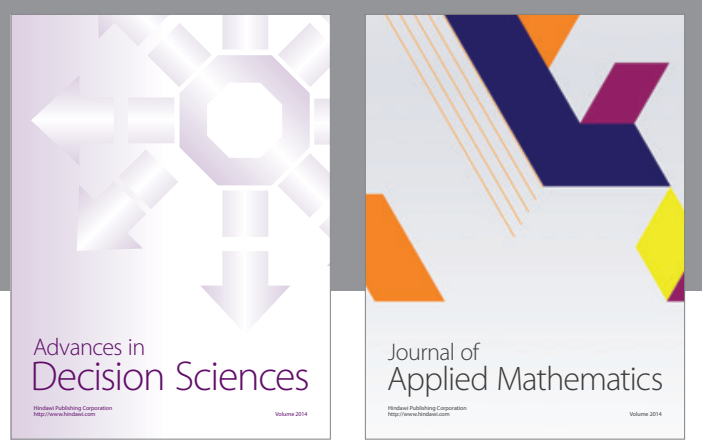

Algebra

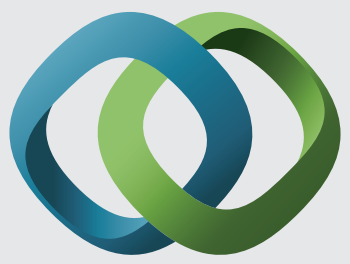

\section{Hindawi}

Submit your manuscripts at

http://www.hindawi.com
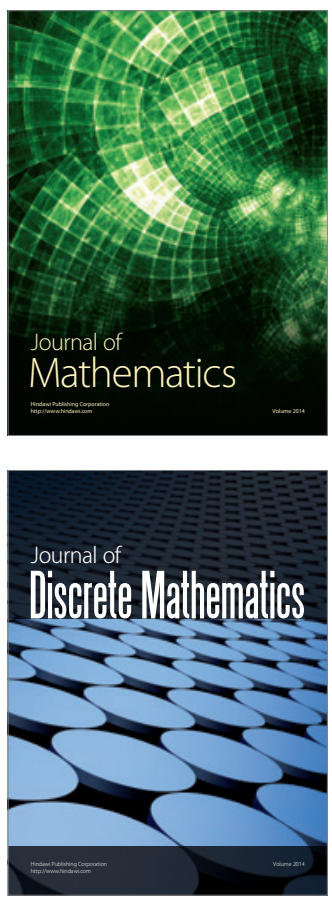

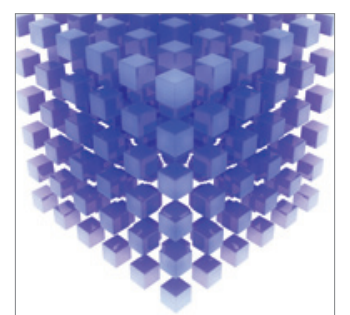

Mathematical Problems in Engineering
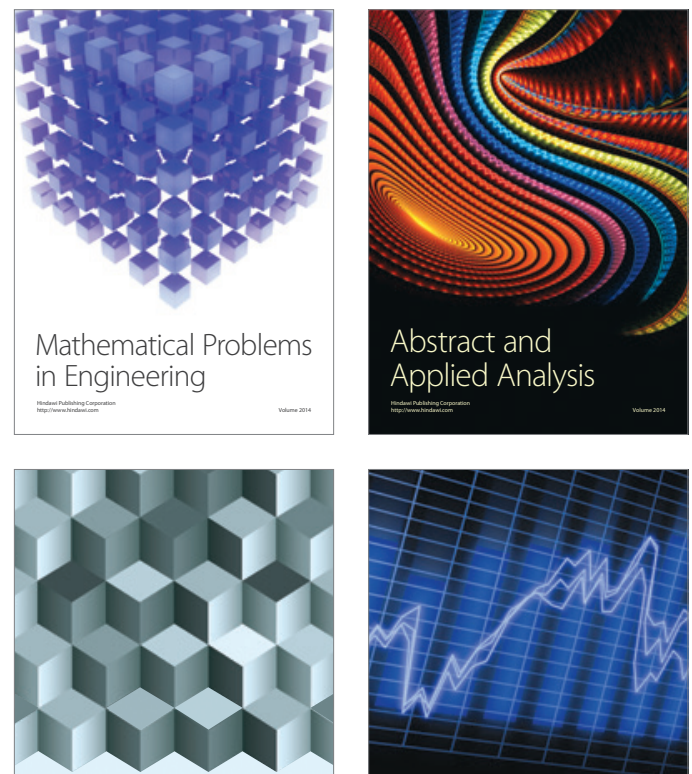

Journal of

Function Spaces

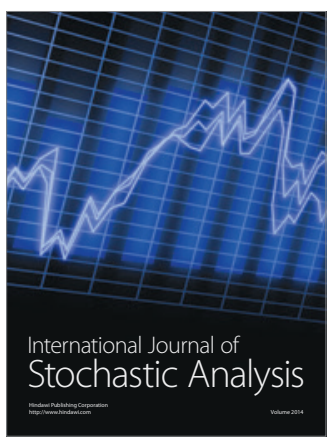

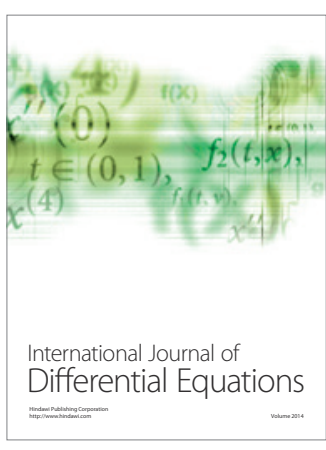
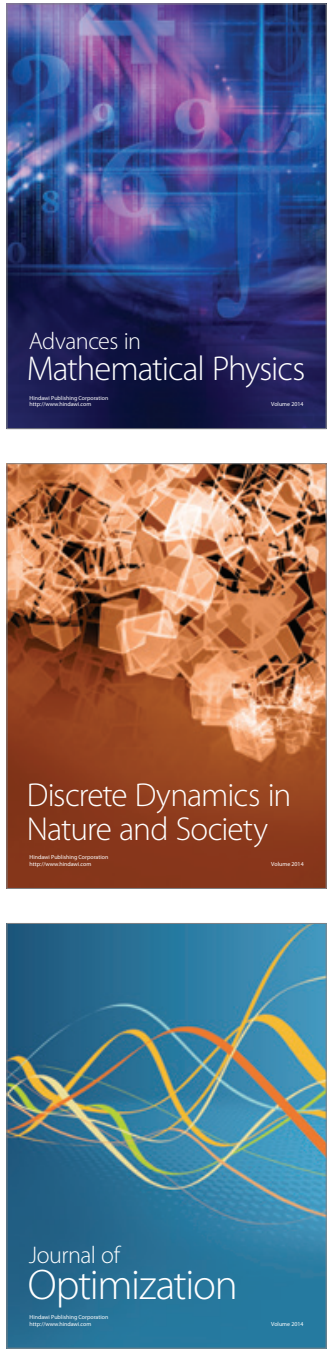